\title{
QSPR Analysis of Degree-Based Topological Indices with physical properties of Benzenoid Hydrocarbons
}

\author{
${ }^{1}$ Rachanna Kanabur, ${ }^{2}$ Vijayalaxmi Shigehalli \\ ${ }^{1}$ Research Scholar, Department of Mathematics \\ Rani Channamma University, Belagavi-591156 \\ Karnataka, India \\ E-mail: rachukanabur@gmail.com \\ ${ }^{2}$ Professor, Department of Mathematics \\ Rani Channamma University, Belagavi-591156 \\ Karnataka, India \\ E-mail: shigehallivs@yahoo.co.in
}

\begin{abstract}
Benzenoid hydrocarbons are condensed polycyclic unsaturated fully conjugated hydrocarbons composed exclusively of six membered rings. Benzenoid system may be represented by different kinds of graphs. Each hexagon of a benzenoid or coronoid system may be represented by a single vertex. In this paper, we find the values of six important degree-based topological indices of molecular graph of benzenoid hydrocarbons. Further, we show that these parameters are highly correlated with physical properties of benzenoid hydrocarbons.
\end{abstract}

Keywords: Degree-based topological indices, Benzenoid hydrocarbons.

\section{Introduction}

Let $G=(V, E)$ be a graph with $\mathrm{n}$ vertices and m edges. The degree of a vertex $u \in V(G)$ is denoted by $d_{G}(u)$ and is the number of vertices that are adjacent to $u$. The edge connecting the vertices $u$ and $v$ is denoted by $u v[6]$.

Chemical graph theory is the branch of mathematical chemistry. It is concerned with handling chemical graphs that represent chemical system. Hence chemical graph theory deals with analysis of all consequences of connectivity in a chemical system. It has found to be a useful tool in QSAR (Quantitative Structure-Activity Relationship) and QSPR (Quantitative Structure-Property Relationship) [8, 10, and 13]. Numerous studies have been made relating to the above mentioned fields by using what are called topological indices. In 1975, Randic [11] proposed a topological index that has become one of the most widely used in both QSAR and QSPR studies. 
It has remarked that mathematics is a more effective tool in the natural sciences than might be reasonably expected. Much of the current panorama of chemical theory has been created on foundations that are essentially graph-theoretical in nature. Chemical graphs are now being used for much different purpose in all the major branches in chemistry [1]. Recent studies have shown that the revived resonance theory successfully gives not only quantitative predictions on $\pi$-electronic properties of benzenoid hydrocarbons [17]. Graph theory is used to mathematically model molecules in order to gain insight into the physical properties of these chemical compounds. Some physical properties, such as the boiling point, are related to the geometric structure of the compound.

Benzenoid hydrocarbons are conveniently represented through benzenoid graphs made up of hexagons in whereas only two types of vertices appear, that is, vertices with degree 2 and vertices with degree $3[2,12]$. This generates only three types of edges in benzenoids, that is, 22, 23 and 33 edges where the numerals are the degrees of vertices making up a given edge.

\section{Selected Degree Based Topological Indices}

We consider the following most studied degree based topological indices for QSPR study.

Randic Index: Historically, the first vertex-degree-based structure descriptors were the graph invariants that now a days are called Zagreb indices however, initially these were intended to be used for a completely different purpose and these were included among topological indices much later. The first genuine degree-based topological index was put-forward in 1975 by Milan Randic in his seminar paper "on characterization of molecular branching [11]" his index was defined as

$$
R(G)=\sum_{u v \in E(G)} \frac{1}{\sqrt{d_{G}(u) d_{G}(v)}}
$$

Harmonic Index: In the 1980s, Siemion Fajtlowicz [18] created computer program for automatic generation of conjectures in graph theory. Then he examined the possible relations between countless graph invariants, among which there was a vertex-degree-based quantity.

$$
H(G)=\sum_{u v \in E(G)} \frac{2}{d_{G}(u)+d_{G}(v)}
$$

Geometric-Arithmetic Index: Another recently conceived vertex-degree based topological index utilizes the difference between the Geometric and Arithmetic means and is defined as

$$
G A(G)=\sum_{u v \in E(G)} \frac{2 \sqrt{d_{G}(u) d_{G}(v)}}{d_{G}(u)+d_{G}(v)}
$$

Where of course, $\sqrt{d_{G}(u) d_{G}(v)}$ and $\frac{2}{d_{G}(u)+d_{G}(v)}$ are the Geometric and Arithmetic means respectively of the degrees of the end vertices of an edge. Recall that the former is always less than or equal to the later. The index was invented by Vukicevic [5] and was named "GeometricArithmetic index". 
Arithmetic-Geometric $\left(\boldsymbol{A} \boldsymbol{G}_{\boldsymbol{I}}\right)$ Index: Let $G=(V, E)$ be a molecular graph, and $d_{G}(u)$ is the degree of the vertex $u$, then $A G_{l}$ index $[29,32]$ of $G$ is defined as

$$
A G_{l}(G)=\sum_{u, v \in E(G)} \frac{d_{G}(u)+d_{G}(v)}{2 \sqrt{d_{G}(u) \cdot d_{G}(v)}}
$$

Where, $A G_{1}$ index is considered for distinct vertices.

The above equation is the sum of the ratio of the Arithmetic mean and Geometric mean of $u$ and $v$, where $d_{G}(u)$ (or $d_{G}(v)$ ) denote the degree of the vertex $u$ (or $v$ ).

SK Index: The $S K$ index $[29,32]$ of a graph $G=(V, E)$ is defined as

$$
S K(G)=\sum_{u, v \in E(G)} \frac{d_{G}(u)+d_{G}(v)}{2}
$$

Where $d_{G}(u)$ and $d_{G}(v)$ are the degrees of the vertices $u$ and $v$ in $G$.

Sum Connectivity Index: The so called "sum connectivity index" is a recent invention by Bo Zhou and Nenad Trinajstic [3]. They noticed that in the definition of Randic's branching index, there is no a prior reason for using the product $d_{G}(u) \times d_{G}(v)$ of vertex degrees, and this term may be replaced by the $\operatorname{sum} d_{G}(u)+d_{G}(v)$. If so, then instead of Randic Index, one gets

$$
S C I(G)=\sum_{u v \in(G)} \frac{1}{\sqrt{d_{G}(u)+d_{G}(v)}}
$$

For recent work on degree-based topological indices, we refer the interested reader to the articles [7, $19,24,28,30,31$ and 33].

\section{Data Sources and Analytical Method}

Energy of benzenoid hydrocarbons are taken from [4,9]. Eight physicochemical properties of benenoid hydrocarbons have been selected on the availability of a suitable body of data: boiling point $(B P)$, critical temperature $(C T)$, critical pressure $(C P)$, critical volume $(C V), G E, \log P, M R$, Henry's law. The values are compiled in Table 2. 
1)

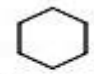

Benzene

2)<smiles>C1CCC2CCCCC2C1</smiles>
Naphthalene

3)<smiles>C1CCC2CC3CCCCC3CC2C1</smiles>
Anthracene

4)<smiles>C1CCC2C(C1)CCC1CCCCC12</smiles>
Phenanthrocene

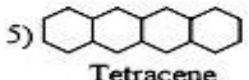
Tetracene

6)<smiles>C1CC2CCCC3CCCC(C1)C23</smiles>

7)

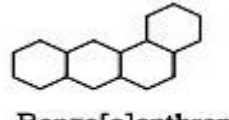

8)<smiles>CC1CCCC2C1CC1C3CCCCC3CCC21</smiles>

9)

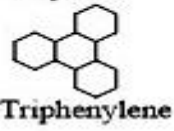

10)

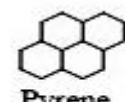

11)

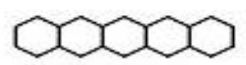

Pentacene

12)

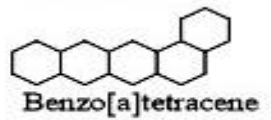

Benzo[c]phenanthrene

13)<smiles></smiles>
Dibenzo[a,h]anthracene

14)<smiles>C1CCC2CC3C(CC2C1)CC1CCCC13</smiles>

Dibenzo $[\mathrm{a}, \mathrm{j}]$ anthracene

15)

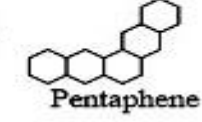

16)<smiles></smiles>

Benzo[g]chrysene

17)

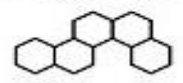

Benzo[c]chrysene

18)

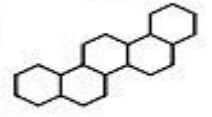

Picene

19)

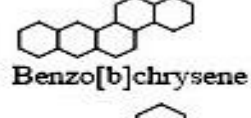

20)

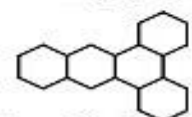

Dibenzo[a,c] anthracene

21)

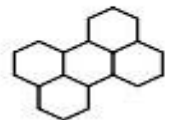

Perylene

22)

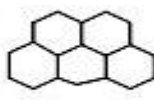

Benzo[e]pyrene

23)

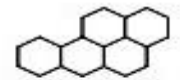

Benzo[a]pyrene
24)

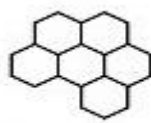

Benzo[ghi]peryl ene

25)

Hexacene

26)

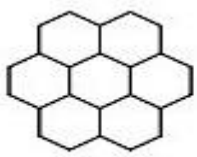

Coronene

Fig.1. Molecular Graphs of benzenoid hydrocarbons 


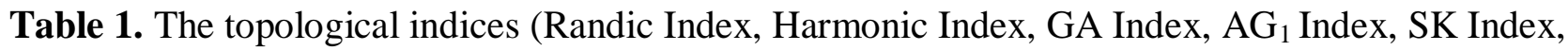
and Sum Connectivity Index) values of benzenoid hydrocarbons.

\begin{tabular}{|c|c|c|c|c|c|c|c|}
\hline S.NO. & Compound Name & $\mathbf{R}(\mathbf{G})$ & $\mathbf{H}(\mathbf{G})$ & GA(G) & $\mathbf{A G}_{\mathbf{1}}(\mathbf{G})$ & SK(G) & SCI(G) \\
\hline 1 & Benzene & 3 & 3 & 6 & 6 & 12 & 3 \\
\hline 2 & Naphthalene & 4.9663 & 4.9333 & 10.9191 & 11.09 & 25 & 5.1971 \\
\hline 3 & Anthracene & 6.9326 & 6.8666 & 15.8383 & 16.19 & 38 & 7.3942 \\
\hline 4 & Phenanthrene & 6.9494 & 6.8999 & 15.8787 & 16.19 & 38 & 7.4080 \\
\hline 5 & Tetracene & 8.8989 & 8.7999 & 20.7575 & 21.29 & 51 & 9.5913 \\
\hline 6 & Benzo[c]phenanthrene & 8.9326 & 8.8666 & 20.8383 & 21.29 & 51 & 9.6189 \\
\hline 7 & Benzoa[a]anthrene & 8.9158 & 8.8333 & 20.7979 & 21.29 & 51 & 9.6051 \\
\hline 8 & Chrysene & 8.9326 & 8.8666 & 20.8383 & 21.29 & 51 & 9.6189 \\
\hline 9 & Triphenylene & 8.9494 & 8.8999 & 20.8787 & 21.14 & 51 & 9.6327 \\
\hline 10 & Pyrene & 7.9326 & 7.8666 & 18.8383 & 18.17 & 44.5 & 8.6189 \\
\hline 11 & Pentacene & 10.8653 & 10.7333 & 25.6767 & 26.39 & 64 & 11.7884 \\
\hline 12 & Benzo[a]tetracene & 10.8821 & 10.7666 & 25.7171 & 26.39 & 64 & 11.8022 \\
\hline 13 & Dibenzo[a,h]anthracene & 10.8989 & 10.7999 & 25.7575 & 26.39 & 64 & 11.8160 \\
\hline 14 & Dibenzo[a,j]anthracene & 10.8989 & 10.7999 & 25.7575 & 26.39 & 64 & 11.8160 \\
\hline 15 & Pentaphene & 10.8821 & 10.7666 & 25.7171 & 26.39 & 64 & 11.8022 \\
\hline 16 & Benzo[g]chrysene & 10.9326 & 10.8666 & 25.8383 & 26.19 & 64 & 11.8436 \\
\hline 17 & Benzo[c]chrysene & 10.9158 & 10.8333 & 25.7979 & 26.39 & 64 & 11.8298 \\
\hline 18 & Picene & 10.9158 & 10.8333 & 25.7979 & 26.39 & 64 & 11.8298 \\
\hline 19 & Benzo[b]chrysene & 10.8989 & 10.7999 & 25.7575 & 26.39 & 64 & 11.8160 \\
\hline 20 & Dibenzo[a,c]anthracene & 10.9158 & 10.8333 & 25.7979 & 26.24 & 64 & 11.8298 \\
\hline 21 & Perylene & 9.9326 & 9.8666 & 23.8383 & 24.19 & 60 & 10.8436 \\
\hline 22 & Benzo[e]pyrene & 9.9326 & 9.8666 & 23.8383 & 24.19 & 60 & 10.8436 \\
\hline 23 & Benzo[a]pyrene & 9.9158 & 9.8333 & 27.7979 & 24.24 & 60 & 10.8298 \\
\hline 24 & Benzo[ghi]perylene & 10.9158 & 10.8333 & 26.7979 & 27.24 & 69 & 12.0546 \\
\hline 25 & Hexacene & 12.8316 & 12.6666 & 30.5959 & 31.29 & 77 & 13.9855 \\
\hline 26 & Coronene & 11.8989 & 11.7999 & 29.7575 & 30.29 & 78 & 13.2655 \\
\hline
\end{tabular}


QSPR Analysis of Degree-Based Topological Indices with physical properties of Benzenoid Hydrocarbons

Table 2. The physical and chemical properties (BP, CT, CP, CV, GE, LogP, MR, Henry's Law) of benzenoid hydrocarbons.

\begin{tabular}{|c|c|c|c|c|c|c|c|c|c|}
\hline $\begin{array}{c}\text { S.N } \\
\text { O. }\end{array}$ & Compound Name & $\begin{array}{l}b p \\
{ }^{\circ} \mathrm{C}\end{array}$ & $C T$ & $\boldsymbol{C P}$ & $C V$ & $G E$ & $\begin{array}{c}\log \\
P\end{array}$ & $M R$ & $\begin{array}{c}\text { Henry } \\
\text { 's } \\
\text { Law }\end{array}$ \\
\hline 1 & Benzene & 80.10 & 323.79 & 47.69 & 263.5 & 121.68 & 2.03 & 25.28 & 0.66 \\
\hline 2 & Naphthalene & 218 & 484.95 & 38.97 & 409.5 & 252.38 & 3.03 & 42.45 & 1.67 \\
\hline 3 & Anthracene & 340 & 586.11 & 32.43 & 555.5 & 383.08 & 4.03 & 59.62 & 2.68 \\
\hline 4 & Phenanthrene & 340 & 586.11 & 32.43 & 555.5 & 383.08 & 4.03 & 59.62 & 2.68 \\
\hline 5 & Tetracene & $\begin{array}{l}432.0 \\
2\end{array}$ & 650.8 & 27.41 & 701.5 & 513.78 & 5.9 & 76.79 & 3.69 \\
\hline 6 & Benzo[c]phenanthrene & 436.7 & 650.8 & 27.41 & 701.5 & 513.78 & 5.03 & 76.79 & 3.69 \\
\hline 7 & Benzoa[a]anthrene & 438 & 650.8 & 27.41 & 701.5 & 513.78 & 5.03 & 76.79 & 3.69 \\
\hline 8 & Chrysene & 448 & 650.8 & 27.41 & 701.5 & 513.78 & 5.03 & 76.79 & 3.69 \\
\hline 9 & Triphenylene & 438 & 650.8 & 27.41 & 701.5 & 513.78 & 5.03 & 76.79 & 3.69 \\
\hline 10 & Pyrene & 404 & 625.65 & 30.73 & 619.5 & 491.18 & 5.08 & 68.36 & 3.47 \\
\hline 11 & Pentacene & 524.6 & 714.53 & 23.47 & 847.5 & 644.48 & 6.02 & 93.96 & 4.7 \\
\hline 12 & Benzo[a]tetracene & 547.5 & 714.53 & 23.47 & 847.5 & 644.48 & 6.02 & 93.96 & 4.7 \\
\hline 13 & Dibenzo[a,h]anthracene & 524 & 714.53 & 23.47 & 847.5 & 644.48 & 6.02 & 93.96 & 4.7 \\
\hline 14 & Dibenzo[a,j]anthracene & 524.7 & 714.53 & 23.47 & 847.5 & 644.48 & 6.02 & 93.96 & 4.7 \\
\hline 15 & Pentaphene & 547.5 & 714.53 & 23.47 & 847.5 & 644.48 & 6.02 & 93.96 & 4.7 \\
\hline 16 & Benzo[g]chrysene & 525 & 714.53 & 23.47 & 847.5 & 644.48 & 6.02 & 93.96 & 4.7 \\
\hline 17 & Benzo[c]chrysene & 524.7 & 714.53 & 23.47 & 847.5 & 644.48 & 6.02 & 93.96 & 4.7 \\
\hline 18 & Picene & 520 & 714.53 & 23.47 & 847.5 & 644.48 & 6.02 & 93.96 & 4.7 \\
\hline 19 & Benzo[b]chrysene & 524.7 & 714.53 & 23.47 & 847.5 & 644.48 & 6.02 & 93.96 & 4.7 \\
\hline 20 & Dibenzo[a,c]anthracene & 518 & 714.53 & 23.47 & 847.5 & 644.48 & 6.02 & 93.96 & 4.7 \\
\hline 21 & Perylene & 400 & 689.17 & 26.08 & 765.5 & 621.88 & 5.34 & 85.53 & 4.48 \\
\hline 22 & Benzo[e]pyrene & 467.5 & 689.17 & 26.08 & 765.5 & 621.88 & 5.34 & 85.53 & 4.48 \\
\hline 23 & Benzo[a]pyrene & 495 & 689.17 & 26.08 & 765.5 & 621.88 & 5.34 & 85.53 & 4.48 \\
\hline 24 & Benzo[ghi]perylene & 500 & 728.06 & 24.85 & 829.5 & 729.98 & 5.66 & 94.28 & 5.27 \\
\hline 25 & Hexacene & 604 & 779.67 & 20.33 & 993.5 & 775.18 & 7.02 & 111.13 & 5.71 \\
\hline 26 & Coronene & 525 & 767.68 & 23.7 & 893.5 & 838.08 & 5.98 & 103.02 & 6.06 \\
\hline
\end{tabular}


Table 3. Correlation of topological indices with physicochemical properties of benzenoid hydrocarbons.

\begin{tabular}{|c|c|c|c|c|c|c|c|c|}
\hline & $\begin{array}{c}\boldsymbol{b} \boldsymbol{C} \\
{ }^{\mathbf{0}} \mathbf{C}\end{array}$ & & $\boldsymbol{C T}$ & $\boldsymbol{C V}$ & $\boldsymbol{G E}$ & $\boldsymbol{L o g} \boldsymbol{P}$ & $\boldsymbol{M R}$ & $\begin{array}{c}\text { Henry's } \\
\text { Law }\end{array}$ \\
\hline$R I$ & 0.975 & 0.979 & -0.977 & $\mathbf{0 . 9 9 9}$ & 0.980 & 0.972 & $\mathbf{1}$ & 0.985 \\
\hline$H I$ & 0.975 & 0.979 & -0.977 & $\mathbf{0 . 9 9 9}$ & 0.980 & 0.972 & $\mathbf{1}$ & 0.985 \\
\hline$G A$ & 0.961 & 0.970 & -0.957 & 0.982 & 0.985 & 0.948 & 0.987 & 0.987 \\
\hline$A G_{1}$ & 0.967 & 0.977 & -0.969 & 0.995 & 0.988 & 0.962 & $\mathbf{0 . 9 9 8}$ & 0.991 \\
\hline$S K$ & 0.955 & 0.975 & -0.957 & 0.987 & 0.996 & 0.950 & 0.994 & $\mathbf{0 . 9 9 8}$ \\
\hline$S C I$ & 0.972 & 0.979 & -0.973 & 0.997 & 0.986 & 0.969 & $\mathbf{1}$ & 0.990 \\
\hline
\end{tabular}

\section{Results and Discussion}

Table 3, the correlation coefficient of topological indices with physicochemical properties of benzenoid hydrocarbons are found to be good.

\subsection{Regression Models:}

We have tested the following linear regression model

$$
\mathrm{P}=\mathrm{A}+\mathrm{B}(\mathrm{TI})
$$

Where, $\mathrm{P}=$ Physical property, $\mathrm{TI}=$ topological index.

Using (7), we have obtained the following different linear models for each degree-based topological index, which are listed below.

1. Randic Index $(R(G))$ :

$b p=0.9431+0.0189 R(G)$

$C T=-5.6908+0.0229 R(G)$

$C P=19.529-0.3693 R(G)$

$C V=-0.5996+0.0136 R(G)$

$G E=1.6679+0.0138 R(G)$

$\log P=-1.0664+1.9883 R(G)$

$M R=0.0979+0.1149 R(G)$

Henry's law $=2.0762+1.8196 R(G)$

\section{Harmonic Index $(H(G))$ :}

$$
\begin{aligned}
& b p=0.9714+0.0187 H(G) \\
& C T=-5.5878+0.0226 H(G) \\
& C P=19.326-0.3648 H(G) \\
& C V=-0.5552+0.0135 H(G)
\end{aligned}
$$


$\mathrm{GE}=1.6793+0.0137 \mathrm{H}(\mathrm{G})$

$\log \mathrm{P}=-1.009+1.9624 \mathrm{H}(\mathrm{G})$

$\mathrm{MR}=0.1325+0.1135 \mathrm{H}(\mathrm{G})$

Henry's law $=2.0833+1.7982 \mathrm{H}(\mathrm{G})$

\section{Geometric-arithmetic Index $(\mathrm{GA}(\mathrm{G}))$ :}

$\mathrm{bp}=0.781+0.0482 \mathrm{GA}(\mathrm{G})$

$\mathrm{CT}=-16.364+0.0586 \mathrm{GA}(\mathrm{G})$

$\mathrm{CP}=47.994-0.9357 \mathrm{GA}(\mathrm{G})$

$\mathrm{CV}=-3.0828+0.0346 \mathrm{GA}(\mathrm{G})$

$\mathrm{GE}=2.2327+0.0359 \mathrm{GA}(\mathrm{G})$

$\log \mathrm{P}=-4.0452+5.0108 \mathrm{GA}(\mathrm{G})$

$\mathrm{MR}=-1.4126+0.2932 \mathrm{GA}(\mathrm{G})$

Henry's law $=3.3398+4.7156 \mathrm{GA}(\mathrm{G})$

4. $\mathrm{AG}_{1}$ Index $\left(\mathrm{AG}_{1}(G)\right)$ :

bp $=0.5954+0.0492 \mathrm{AG}_{1}(\mathrm{G})$

$\mathrm{CT}=-16.959+0.0599 \mathrm{AG}_{1}(\mathrm{G})$

$\mathrm{CP}=48.96-0.9613 \mathrm{AG}_{1}(\mathrm{G})$

$\mathrm{CV}=-3.5531+0.0356 \mathrm{AG}_{1}(\mathrm{G})$

$\mathrm{GE}=2.1308+0.0366 \mathrm{AG}_{1}(\mathrm{G})$

$\log \mathrm{P}=-4.5821+5.1622 \mathrm{AG}_{1}(\mathrm{G})$

$\mathrm{MR}=-1.7856+0.301 \mathrm{AG}_{1}(\mathrm{G})$

Henry's law $=3.2362+4.8071 \mathrm{AG}_{1}(\mathrm{G})$

\section{SK Index (SK (G)) :}

$\mathrm{bp}=-1.8424+0.127 \mathrm{SK}(\mathrm{G})$

$\mathrm{CT}=-48.149+0.1516 \mathrm{SK}(\mathrm{G})$

$\mathrm{CP}=122.9-2.4803 \mathrm{SK}(\mathrm{G})$

$\mathrm{CV}=-12.87+0.0923 \mathrm{SK}(\mathrm{G})$

$\mathrm{GE}=0.9974+0.0963 \mathrm{SK}(\mathrm{G})$

$\log \mathrm{P}=-15.208+13.314 \mathrm{SK}(\mathrm{G})$

$\mathrm{MR}=-8.4873+0.7823 \mathrm{SK}(\mathrm{G})$

Henry's law $=3.9951+12.631 \mathrm{SK}(\mathrm{G})$

\section{Sum connectivity Index (SCI (G)) :}

$$
\begin{aligned}
& \mathrm{bp}=0.7035+0.0212 \mathrm{SCI}(\mathrm{G}) \\
& \mathrm{CT}=-6.8131+0.0258 \mathrm{SCI}(\mathrm{G}) \\
& \mathrm{CP}=21.532+0.4139 \mathrm{SCI}(\mathrm{G}) \\
& \mathrm{CV}=-1.0492+0.0153 \mathrm{SCI}(\mathrm{G}) \\
& \mathrm{GE}=1.4258+0.0157 \mathrm{SCI}(\mathrm{G}) \\
& \mathrm{LogP}=-1.5481+2.2281 \mathrm{SCI}(\mathrm{G}) \\
& \mathrm{MR}=-0.2823+0.1292 \mathrm{SCI}(\mathrm{G}) \\
& \text { Henry's law }=1.8954+2.0581 \mathrm{SCI}(\mathrm{G})
\end{aligned}
$$


Table 4. Statistical parameters for the linear QSPR model for $R(G)$.

\begin{tabular}{|l|l|l|l|l|l|l|l|}
\hline Physical Properties & $\mathbf{N}$ & $\mathbf{a}$ & $\mathbf{b}$ & $\mathbf{r}$ & $\mathbf{R}^{2}$ & $\mathbf{S}$ & $\mathbf{F}$ \\
\hline Boiling point & 26 & 0.9431 & 0.0189 & 0.975 & 0.951 & 0.490 & 470.31 \\
\hline Critical temperature & 26 & -5.6908 & 0.0229 & 0.979 & 0.958 & 0.458 & 542.78 \\
\hline Critical Pressure & 26 & 19.529 & -0.3693 & -0.977 & -0.954 & 0.475 & 502.1 \\
\hline Critical volume & 26 & -0.5996 & 0.0136 & $\mathbf{0 . 9 9 9}$ & 0.998 & 0.098 & 1.225 \\
\hline GE & 26 & 1.6679 & 0.0138 & 0.980 & 0.961 & 0.442 & 583.65 \\
\hline LogP & 26 & -1.0664 & 1.9883 & 0.972 & 0.946 & 0.518 & 418.37 \\
\hline MR & 26 & 0.0979 & 0.1149 & $\mathbf{1}$ & 1 & 0.019 & 3.061 \\
\hline Henry's law & 26 & 2.0762 & 1.8196 & 0.985 & 0.975 & 0.385 & 776.62 \\
\hline
\end{tabular}

Table 5. Statistical parameters for the linear QSPR model for $H(G)$.

\begin{tabular}{|l|c|c|c|c|c|c|c|}
\hline Physical Properties & $\mathbf{N}$ & $\mathbf{a}$ & $\mathbf{b}$ & $\mathbf{R}$ & $\mathbf{R}^{2}$ & $\mathbf{S}$ & $\mathbf{F}$ \\
\hline Boiling point & 26 & 0.9714 & 0.0187 & 0.975 & 0.950 & 0.489 & 459.89 \\
\hline Critical temperature & 26 & -5.5878 & 0.0226 & 0.979 & 0.958 & 0.451 & 544.33 \\
\hline Critical Pressure & 26 & 19.326 & -0.3648 & -0.977 & 0.954 & 0.470 & 500.56 \\
\hline Critical volume & 26 & -0.5552 & 0.0135 & $\mathbf{0 . 9 9 9}$ & 0.998 & 0.107 & 1.005 \\
\hline GE & 26 & 1.6793 & 0.0137 & 0.980 & 0.961 & 0.432 & 596.17 \\
\hline LogP & 26 & -1.009 & 1.9624 & 0.972 & 0.944 & 0.520 & 404.85 \\
\hline MR & 26 & 0.1325 & 0.1135 & $\mathbf{1}$ & 1 & 0.358 & 9.011 \\
\hline Henry's law & 26 & 2.0833 & 1.7982 & 0.985 & 0.971 & 0.376 & 794.96 \\
\hline
\end{tabular}


QSPR Analysis of Degree-Based Topological Indices with physical properties of Benzenoid Hydrocarbons

Table 6. Statistical parameters for the linear QSPR model for $G A(G)$.

\begin{tabular}{|l|c|c|c|c|c|c|c|}
\hline Physical Properties & $\mathbf{N}$ & $\mathbf{a}$ & $\mathbf{b}$ & $\mathbf{r}$ & $\mathbf{R}^{2}$ & $\mathbf{S}$ & $\mathbf{F}$ \\
\hline Boiling point & 26 & 0.781 & 0.0482 & 0.961 & 0.924 & 1.589 & 290.56 \\
\hline Critical temperature & 26 & -16.364 & 0.0586 & 0.970 & 0.941 & 1.393 & 385.51 \\
\hline Critical Pressure & 26 & 47.994 & -0.9357 & -0.957 & 0.916 & 1.664 & 263.11 \\
\hline Critical volume & 26 & -3.0828 & 0.0346 & 0.982 & 0.964 & 1.092 & 641.53 \\
\hline GE & 26 & 2.2327 & 0.0359 & 0.985 & 0.969 & 1.006 & 760.35 \\
\hline LogP & 26 & -4.0452 & 5.0108 & 0.948 & 0.898 & 1.834 & 212.13 \\
\hline MR & 26 & -1.4126 & 0.2932 & 0.987 & 0.974 & 0.928 & 897.84 \\
\hline Henry's law & 26 & 3.3398 & 4.7156 & 0.987 & 0.974 & 0.921 & 911.29 \\
\hline
\end{tabular}

Table 7. Statistical parameters for the linear QSPR model for $A G_{l}(G)$.

\begin{tabular}{|l|c|c|c|c|c|c|c|}
\hline Physical Properties & $\mathbf{N}$ & $\mathbf{a}$ & $\mathbf{B}$ & $\mathbf{R}$ & $\mathbf{R}^{2}$ & $\mathbf{S}$ & $\mathbf{F}$ \\
\hline Boiling point & 26 & 0.5954 & 0.0492 & 0.967 & 0.935 & 1.493 & 343.10 \\
\hline Critical temperature & 26 & -16.959 & 0.0599 & 0.977 & 0.955 & 1.239 & 508.97 \\
\hline Critical Pressure & 26 & 48.96 & -0.9613 & -0.969 & 0.939 & 1.440 & 370.84 \\
\hline Critical volume & 26 & -3.5531 & 0.0356 & 0.995 & 0.990 & 0.570 & 2.496 \\
\hline GE & 26 & 2.1308 & 0.0366 & 0.988 & 0.976 & 0.907 & 970.65 \\
\hline LogP & 26 & -4.5821 & 5.1622 & 0.962 & 0.926 & 1.592 & 299.04 \\
\hline MR & 26 & -1.7856 & 0.301 & $\mathbf{0 . 9 9 8}$ & 0.997 & 0.329 & 7.530 \\
\hline Henry's law & 26 & 3.2362 & 4.8071 & 0.991 & 0.983 & 0.761 & 1.389 \\
\hline
\end{tabular}


Table 8. Statistical parameters for the linear QSPR model for $S K(G)$.

\begin{tabular}{|l|c|c|c|c|c|c|c|}
\hline Physical Properties & $\mathbf{N}$ & $\mathbf{a}$ & $\mathbf{b}$ & $\mathbf{R}$ & $\mathbf{R}^{2}$ & $\mathbf{S}$ & $\mathbf{F}$ \\
\hline Boiling point & 26 & -1.8424 & 0.127 & 0.955 & 0.911 & 4.545 & 246.25 \\
\hline Critical temperature & 26 & -48.149 & 0.1561 & 0.975 & 0.950 & 3.412 & 455.56 \\
\hline Critical Pressure & 26 & 122.9 & -2.4803 & -0.957 & 0.917 & 4.400 & 264.41 \\
\hline Critical volume & 26 & -12.87 & 0.0923 & 0.987 & 0.975 & 2.412 & 935.79 \\
\hline GE & 26 & 0.9974 & 0.0963 & 0.996 & 0.991 & 1.420 & 2.744 \\
\hline LogP & 26 & -15.208 & 13.314 & 0.950 & 0.903 & 4.753 & 233.18 \\
\hline MR & 26 & -8.4873 & 0.7823 & 0.994 & 0.987 & 1.722 & 1.859 \\
\hline Henry's law & 26 & 3.9951 & 12.631 & $\mathbf{0 . 9 9 8}$ & 0.995 & 1.056 & 4.983 \\
\hline
\end{tabular}

Table 9. Statistical parameters for the linear QSPR model for SCI (G).

\begin{tabular}{|l|c|c|c|c|c|c|c|}
\hline Physical Properties & $\mathbf{N}$ & $\mathbf{a}$ & $\mathbf{b}$ & $\mathbf{r}$ & $\mathbf{R}^{2}$ & $\mathbf{S}$ & $\mathbf{F}$ \\
\hline Boiling point & 26 & 0.7035 & 0.0212 & 0.972 & 0.944 & 0.593 & 403.29 \\
\hline Critical temperature & 26 & -6.8131 & 0.0258 & 0.979 & 0.959 & 0.506 & 563 \\
\hline Critical Pressure & 26 & 21.532 & 0.4139 & -0.973 & 0.947 & 0.577 & 427.27 \\
\hline Critical volume & 26 & -1.0492 & 0.0153 & 0.997 & 0.994 & 0.190 & 4.106 \\
\hline GE & 26 & 1.4258 & 0.0157 & 0.986 & 0.972 & 0.417 & 840.38 \\
\hline LogP & 26 & -1.5481 & 2.2281 & 0.969 & 0.938 & 0.623 & 363.73 \\
\hline MR & 26 & -0.2823 & 0.1292 & $\mathbf{1}$ & 0.999 & 0.074 & 2.721 \\
\hline Henry's law & 26 & 1.8954 & 2.0581 & 0.990 & 0.980 & 0.352 & 1.185 \\
\hline
\end{tabular}

By inspection of the data in Tables 4 to 9 , it is possible to draw a number of conclusions for the given topological indices like invariants. 
The QSPR study of Randic index reveals that $R(G)$ can be useful in predicting the boiling point, critical temperature, critical pressure, critical volume, GE, LogP, MR, Henry's law of benzenoid hydrocarbons also from Table 4, one can easily verify that Randic index shows good correlation with all physical properties of benzenoid hydrocarbons. We can see that the correlation coefficient value of $R(G)$ with physical properties of benzenoid hydrocarbons lies between 0.972 to 1 . Further, the correlation coefficient value of Randic index is very high for the MR of benzenoid hydrocarbons with $r=1$.Thus the QSPR study reveals that the Randic index is an useful tool in predicting the physical properties of benzenoid hydrocarbons.

The harmonic index did not attract anybody's attention, especially, not of chemists. No chemical applications of the harmonic index were reported so far, but knowing the present situation in the mathematical chemistry we here explore the chemical applications of harmonic index. Table 5, one can easily verify that Harmonic index shows good correlation with all physical properties of benzenoid hydrocarbons. Further, we can see that the correlation coefficient value of $H(G)$ with physical properties of benzenoid hydrocarbons lies between 0.972 to 1 . Further, the correlation coefficient value of Harmonic index is very high for the $M R$ of benzenoid hydrocarbons with $\mathrm{r}=1$. Thus the QSPR study reveals that the Harmonic index is a useful tool in predicting the physical properties of benzenoid hydrocarbons.

The QSPR study of Geometric-Arithmetic index reveals that GA $(\mathrm{G})$ can be useful in predicting the boiling point, critical temperature, critical pressure, critical volume, GE, LogP, MR, Henry's law of benzenoid hydrocarbons also from Table 6, one can easily verify that Geometric-Arithmetic index shows good correlation with all physical properties of benzenoid hydrocarbons. We can see that the correlation coefficient value of GA (G) with physical properties of benzenoid hydrocarbons lies between 0.961 to 0.987 . Further, the correlation coefficient value of Geometric-Arithmetic index is very high for the Henry's law of benzenoid hydrocarbons with $\mathrm{r}=0.987 \sqcup 1$. Thus the QSPR study reveals that the GeometricArithmetic index is a useful tool in predicting the physical properties of benzenoid hydrocarbons.

Motivated by Geometric-Arithmetic index. Here we introduced a new topological index namely, Arithmetic-Geometric $\left(\mathrm{AG}_{1}\right)$ index. The QSPR study of Arithmetic-Geometric $\left(A G_{1}\right)$ index reveals that $A G_{1}(G)$ can be useful in predicting the boiling point, critical temperature, critical pressure, critical volume, GE, LogP, MR, Henry's law of benzenoid hydrocarbons also from Table 7 , one can easily verify that Arithmetic-Geometric $\left(\mathrm{AG}_{1}\right)$ index shows good correlation with all physical properties of benzenoid hydrocarbons. Further, we can see that the correlation coefficient value of $A_{1}(G)$ with physical properties of benzenoid hydrocarbons lies between 0.962 to 0.998 . Further, the correlation coefficient value of Arithmetic-Geometric $\left(\mathrm{AG}_{1}\right)$ index is very high for the MR of benzenoid hydrocarbons with $\mathrm{r}=0.998 \sqcup 1$. Thus the QSPR study reveals that the ArithmeticGeometric $\left(\mathrm{AG}_{1}\right)$ index is an useful tool in predicting the physical properties of benzenoid hydrocarbons.

Motivated by Harmonic index. Here we introduced a new topological index namely, SK index. The QSPR study of SK index reveals that the predicting power of SK index for the physical properties of benzenoid hydrocarbons is equally good as Arithmetic-Geometric 
$\left(\mathrm{AG}_{1}\right)$ index. Table 8 the correlation coefficient value lies between 0.950 to 0.998 . Surprisingly, we could that the correlation of SK index with Henry's law of benzenoid hydrocarbons is very high with correlation coefficient $r=0.998 \sqcup 1$. Thus SK index found to be useful tool in predicting the physical properties of benzenoid hydrocarbons.

The QSPR study in Table 9 reveals that the predicting power of sum-connectivity index is very high compared to other degree-based topological indices. The correlation coefficient value lies between 0.969 to 1 . Further, the correlation coefficient value of sum-connectivity index is very high for the MR of benzenoid hydrocarbons with $r=1$. Thus the QSPR study reveals that the sum-connectivity index is an useful tool in predicting the physical properties of benzenoid hydrocarbons.

From practical point of view, topological indices for which the absolute values of correlation coefficient are less than 0.8 can be characterized as useless. Thus, the QSPR study of the six degree-based topological indices with physical properties of 26 benzenoid hydrocarbons helps us to characterize useful topological indices with absolute value of correlation coefficient lies between 0.950 to1.
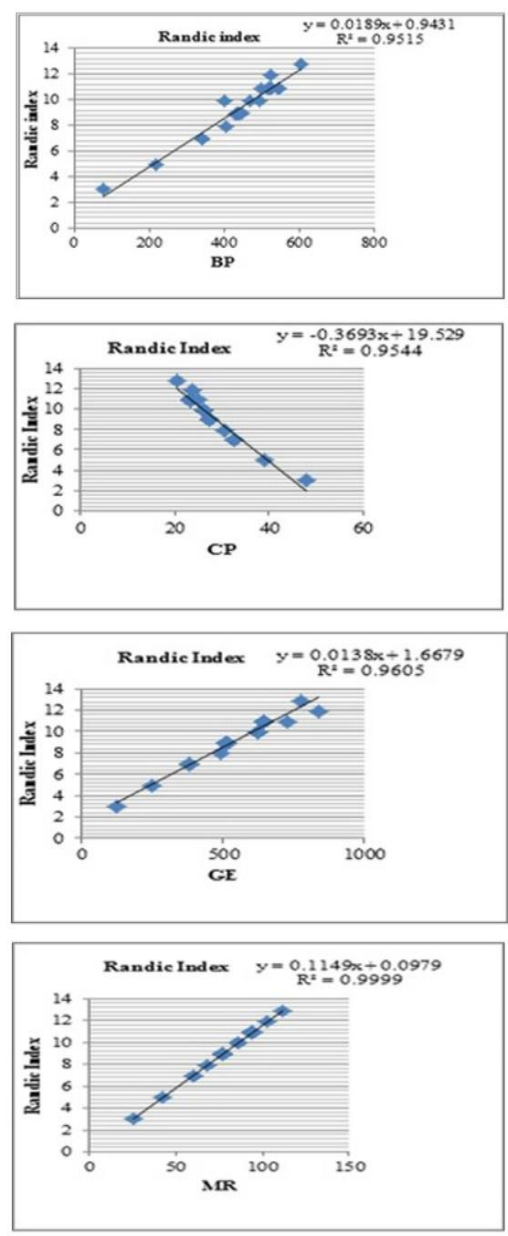
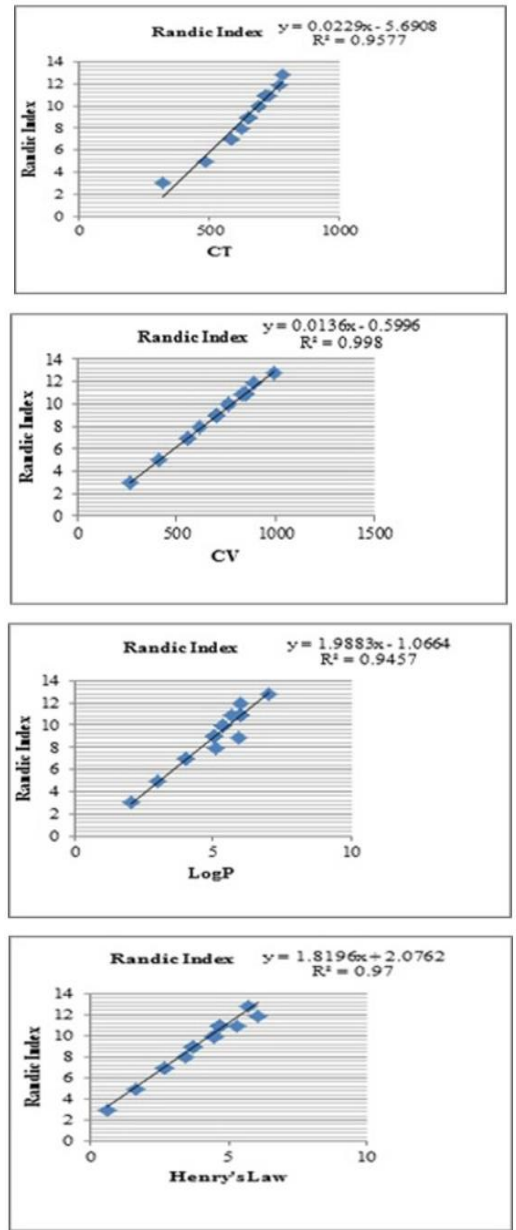
QSPR Analysis of Degree-Based Topological Indices with physical properties of Benzenoid Hydrocarbons

Fig.2. Correlation of Randic index with physicochemical properties of benzenoid hydrocarbons.
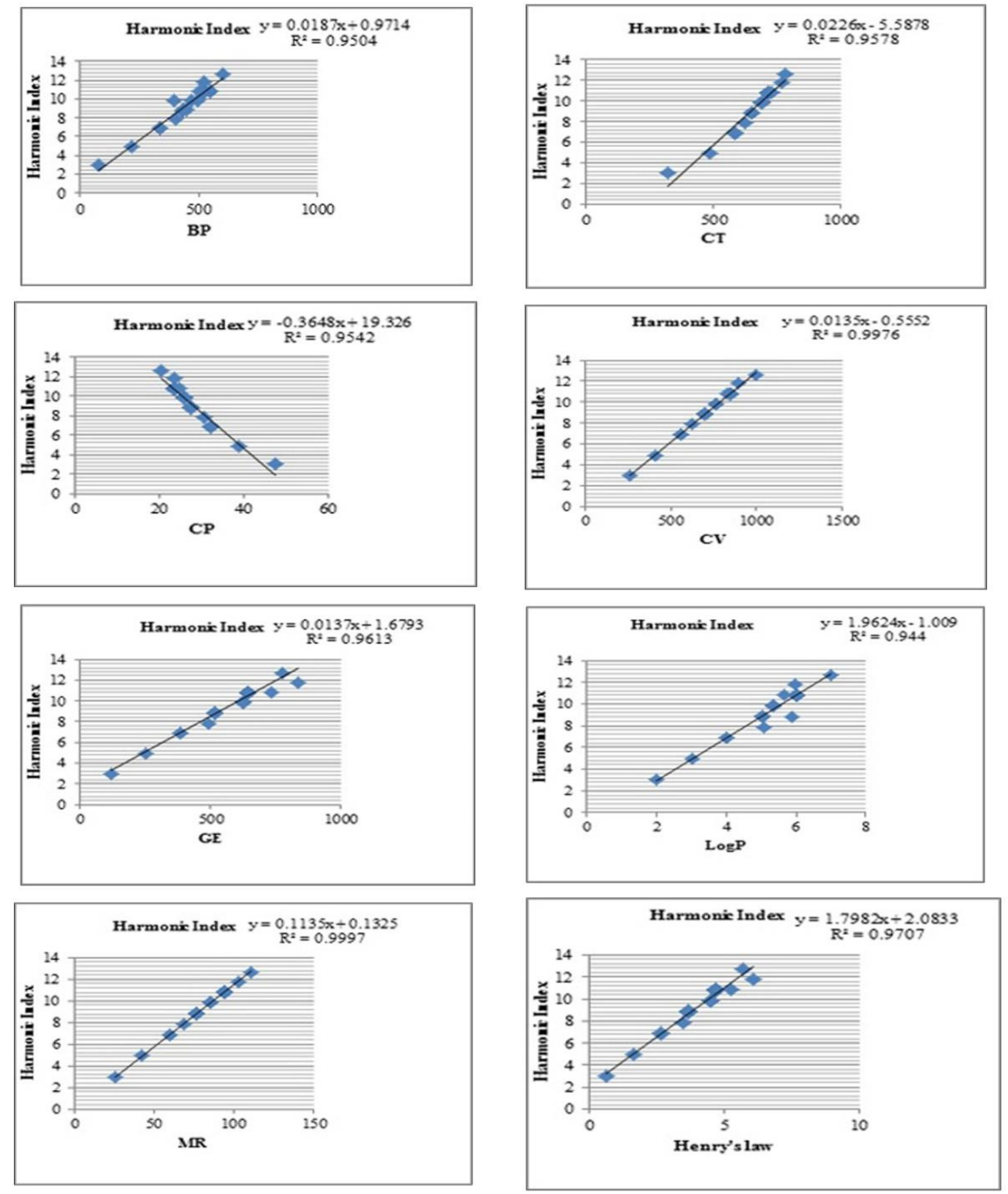

Fig.3. Correlation of Harmonic index with physicochemical properties of benzenoid hydrocarbons. 

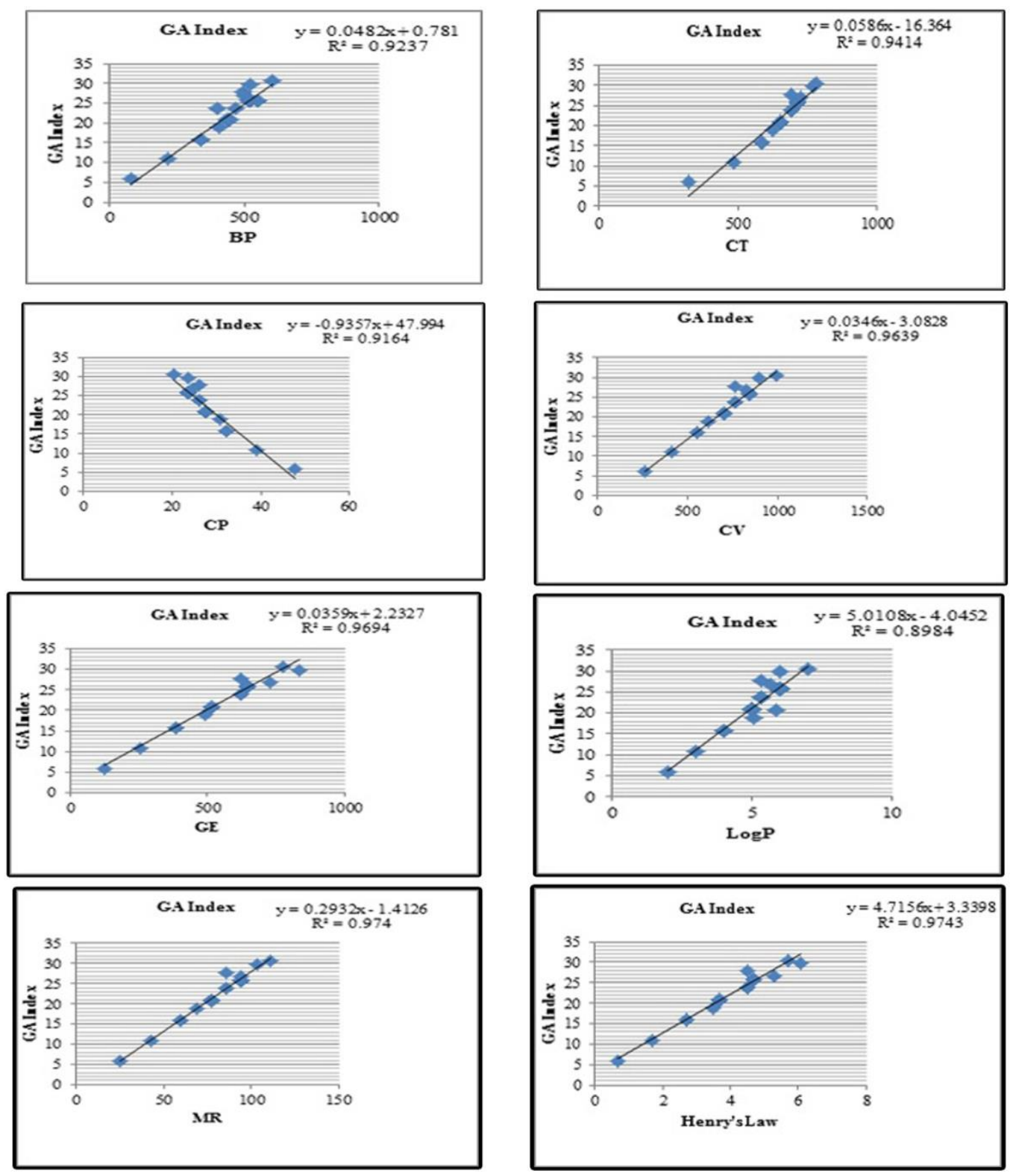

Fig.4. Correlation of Geometric-Arithmetic index with physicochemical properties of benzenoid hydrocarbons. 

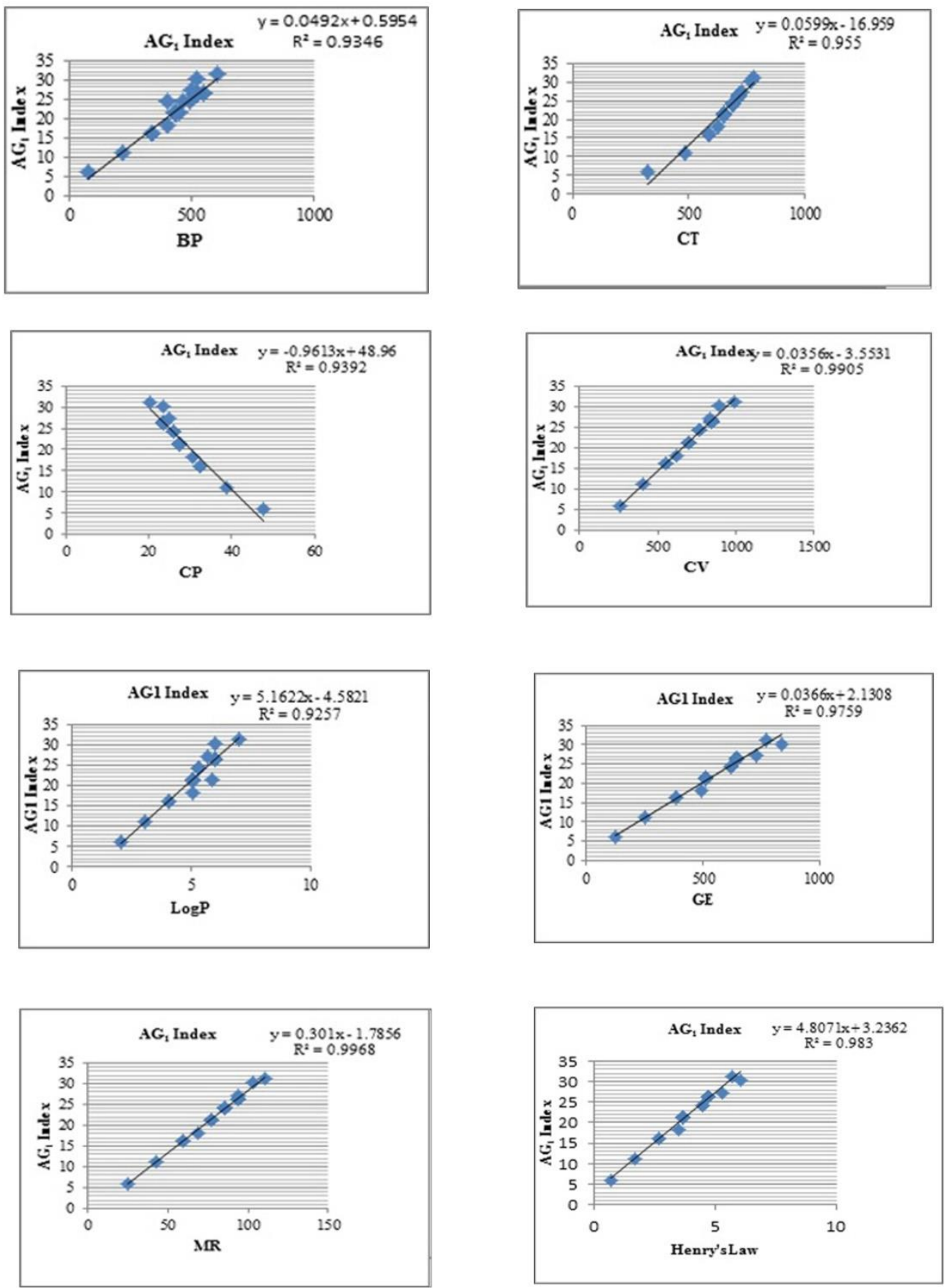

Fig.5. Correlation of $\mathrm{AG}_{1}$ index with physicochemical properties of benzenoid hydrocarbons. 

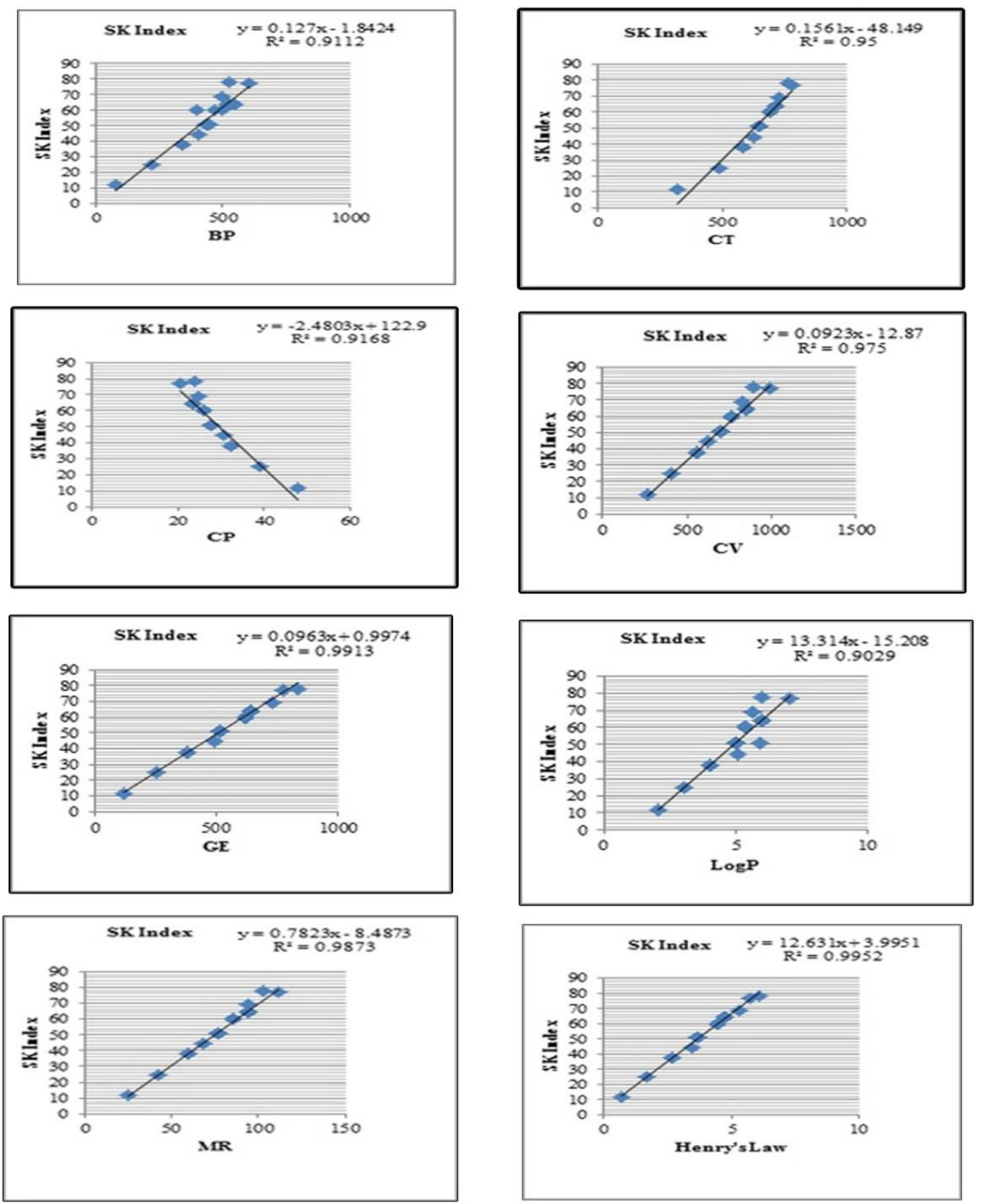

Fig.6. Correlation of SK index with physicochemical properties of benzenoid hydrocarbons. 

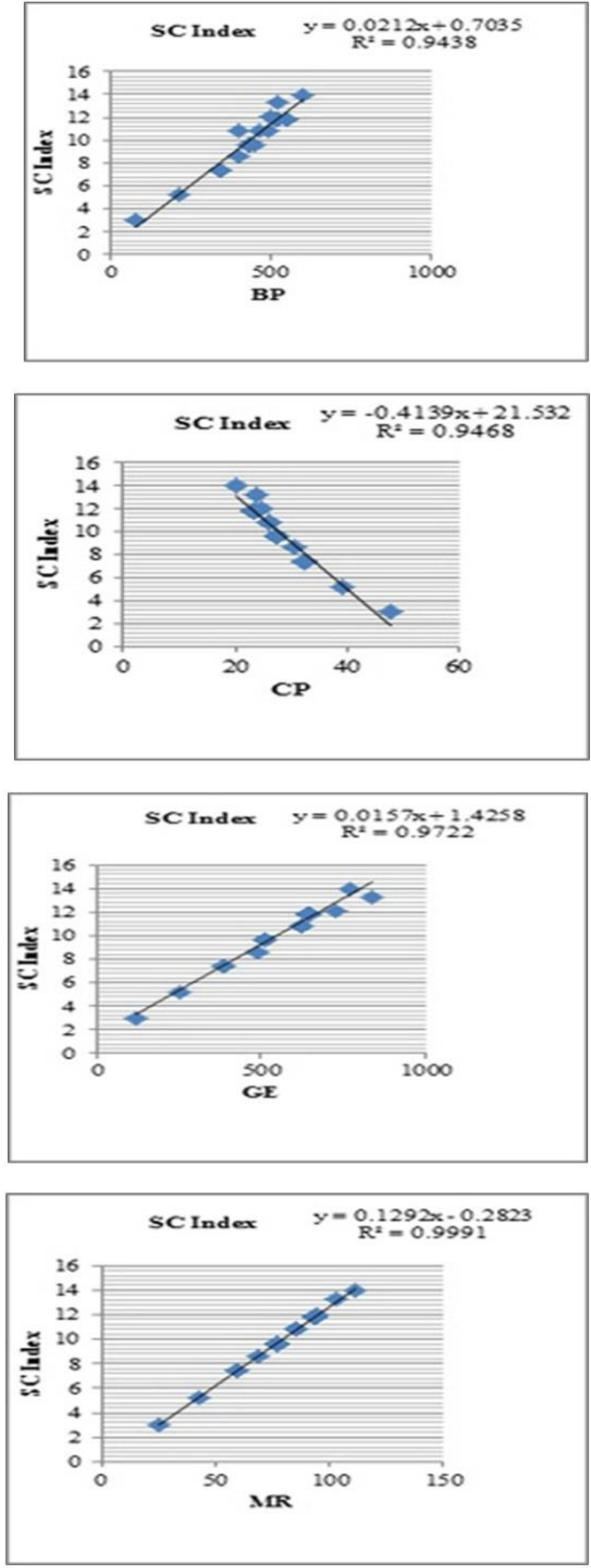
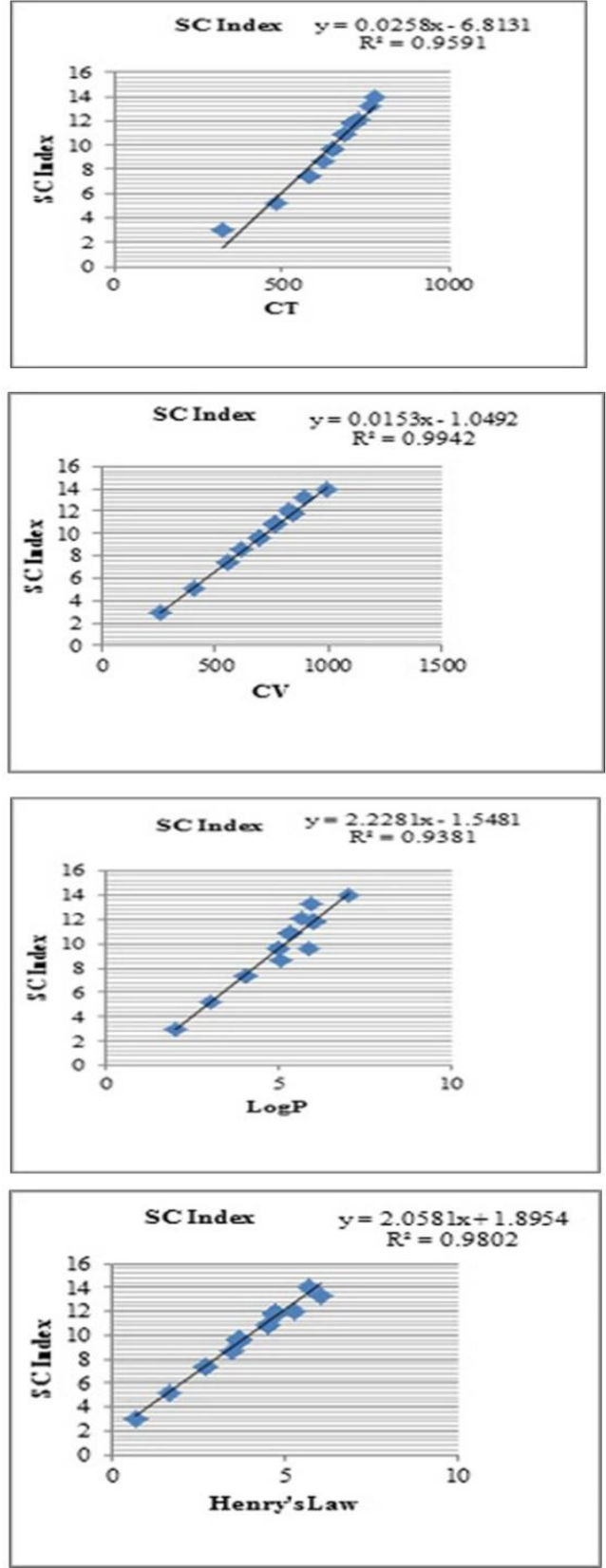

Fig.7. Correlation of Sum-Connectivity index with physicochemical properties of benzenoid hydrocarbons. 


\section{References}

[1] B. Bonchere, D. H. Rouvray, Chemical graph theory introduction and Fundamentals, Gordon and Breach Science Publishers, S. A. O. Favaron, M. Maheo, J.F. Scale, Some eigenvalue properties in graphs(conjectures of Graffiti - II), Discrete Math., 111, (1993), 197220.

[2] B. Lucic, N. Trinajistic, Bo Zhou, Comparison between the sum-connectivity index and product-connectivity index for benzenoid hydrocarbons, Chemical Physics Letters, 475 (2009), 146-148.

[3] B. Zhou, N. Trinajstic, Sum-connectivity index, J. Math. Chem. 46, (2009), 1252.

[4] C. A. Coulson, A. Streitwieser, Dictionary of $\pi$-Electron Calculations, Freeman, San Francisco, 1965.

[5] D.Vukicevic, B.Furtula, Topological index based on the ratios of geometrical and arithmetical mean of end-vertex degrees of edges, J. Math. Chem. 26, (2009), 1369-1376.

[6] F. Harary, Graph theory, Addison-Wesely, Reading mass (1969).

[7] I. Gutman, Degree-based topological indices, Croat. Chem. Acta, 86, (2013), 251-361.

[8] J. Devillers , A. T. Balban, Topological indices and related descriptors in QSAR and QSPR, Gordon and Breach Science Publishers, Amsterdam, Netherland, (1999).

[9] J.R. Dias, Handbook of Polycyclic Hydrocarbons. Part A: Benzenoid Hydrocarbons, Elsevier, Amsterdam, 1987.

[10] M. Randic, on characterization of molecular branching, J. Am. Chem.Soc. 97, (1975), 66096615.

[11] M. Randic, on history of the Randic index and emerging hostility toward chemical graph theory, MATCH Commun. Math. Comput. Chem., 59, (2008), 5-124.

[12] M. Randic, Quantitative Structure-Property Relationship: boiling point and planar benzenoids, New. J. Chem. 20,(1996),1001-1009.

[13] M. Thakur, A. Thakur, P. V. Khadikar, QSAR study of benzene sulphonamide carbonic anhydrase inhibitors: Topological approach using Balban index, Bioorg. Med. Chem. 12(4), (2004), 789-793.

[14] M.V.Diudea, I. Gutman, J.Lorentz, Molecular Topology, Babes-Bolyai University, Romania 2001.

[15] Minati Kuanar, Saroj K Kuanar, Bijay K Mishra, Correlation of line graph parameters with physical properties of octane isomers, Indian Journal of Chemistry, 38A, (1999),525-528.

[16] N.Trinajstic, Chemical Graph theory, CRC Press, Boca Raton (1992).

[17] Noriko Ohkami, Haruo Hosoya, Topological dependency of the aromatic sextets in polycyclic benzenoid hydrocarbons. Recursive relation of the sextet polynomial,Theoret. Chem. Acta (Berl), 64, (1983), 153-170.

[18] S. Fajtlowicz, Harmonic Index, Congr. Number. 60, (1987), 187.

[19] S.M. Hosamani, Deepa. M.Perigidad, ShrutiY. Jamagoud Yallavva B. Maled and Sharada Gavade, QSPR Analysis of Certain Degree Based Topological Indices, Journal of Statistics Applications and Probability,2016(in press).

[20] S.M. Hosamani, I.Gutman, Zagreb indices of transformation graphs and total transformation graphs, Appl.Math.Comput. 247, (2014), 1156-1160.

[21] S.M. Hosamani, M.Krzywkowski, on the difference of Zagreb coindices ofgraph operations, Gulf Journal of Mathematics, 4(3), (2016), 36-41. 
[22] S.M. Hosamani, S.H. Malaghan, I.N. Cangul, The first geometric-arithmetic index of graph operations, Advances and Applications in Mathematical Sciences, 14(6), (2015), 155-163.

[23] S.M.Hosamani, Computing Sanskrit index of certain nanostructures, J.Appl. Math. Comput.1-9, (2016).

[24] S.M.Hosamani, Correlation of Domination Parameters with physico- chemical properties of Octane isomers, Applied Mathematics and Nonlinear Sciences, 1(2), (2016). 345-352.

[25] Simge Öztunç, Ali Mutlu and Necdet Bildik, Computing Hypercrossed Complex Pairings in Digital Images, Abstract and Applied Analysis, 2013.

[26] Simge Öztunç, Necdet Bildik and Ali Mutlu, The Construction of Simplicial Groups in Digital Images, Journal of Inequalities and Applications, 143,(2013), 1-13.

[27] Simge Öztunç, Sultan İhtiyar, Properties of Soft Homotopy in Digital Images, American Instutite of Physics, 1798, 020120 (2017), 1-9.

[28] V.S Shigehalli, R.Kanabur, Degree Based Topological Indices of n-Heptane Isomers, Jamal Academic Research Journal: An Interdisciplinary Special Issue (2016), 235-238.

[29] V.S. Shigehalli, R. Kanabur , New Version of Degree-Based Topological Indices of Certain nanotube, Journal of Mathematical Nano science, 6(1-2), (2016), 29-39.

[30] V.S. Shigehalli, R. Kanabur , Arithmetic-Geometric indices of Path Graph, Journal of Computer and Mathematical sciences, 6(1), (2015), 19-24.

[31] V.S. Shigehalli, R. Kanabur, Arithmetic-Geometric indices of some class of Graph, Journal of Computer and Mathematical sciences, 6(4),(2015), 194-199.

[32] V.S. Shigehalli, R. Kanabur, Computation of New Degree-Based Topological Indices of Graphene, Journal of Mathematics, 2016.

[33] V.S. Shigehalli, R.Kanabur, Correlation between the Arithmetic-Geometric Indices of nheptane Alkane Isomers, IOSR Journal of Mathematics(IOSR-JM),12(2) Ver.IV, (2016), 3639. 